\title{
The Role of Phosphatidic Acid and Cardiolipin in Stability of the Tetrameric Assembly of Potassium Channel KcsA
}

\author{
Mobeen Raja
}

Received: 31 December 2009/ Accepted: 15 March 2010/Published online: 30 March 2010

(C) The Author(s) 2010. This article is published with open access at Springerlink.com

\begin{abstract}
In this study, the roles of two anionic phospholipids-phosphatidic acid (PA), which is an important signaling molecule, and cardiolipin (CL), which plays a crucial role in the bioenergetics of the cell-in stabilizing the oligomeric structure of potassium channel KcsA were determined. The stability of KcsA was drastically increased as a function of PA or CL content ( $\mathrm{mol} \%)$ in phosphatidylcholine (PC) bilayers. Deletion of the membrane-associated $\mathrm{N}$ terminus significantly reduced channel stability at high levels of PA content; however, the intrinsic stability of this protein was marginally affected in the presence of CL. These studies indicate that the electrostatic-hydrogen bond switch between PA and $\mathrm{N}$ terminus, involving basic residues, is much stronger than the stabilizing effect of CL. Furthermore, the unique properties of the PA headgroup alter protein assembly and folding properties differently from the CL headgroup, and both lipids stabilize the tetrameric assembly via their specific interaction on the extraor the intracellular side of KcsA.
\end{abstract}

Keywords Potassium channel KcsA .

Tetrameric stability - Anionic lipids · Phosphatidic acid . Cardiolipin · N-terminus - Electrostatic interactions ·

Protein-lipid interaction

\footnotetext{
M. Raja

Department of Biochemistry of Membranes,

Center for Biomembranes and Lipid Enzymology,

Institute of Biomembranes, Utrecht University,

Padualaan 8, $3584 \mathrm{CH}$ Utrecht, The Netherlands

M. Raja ( ()

School of Molecular and Systems Medicine, Alberta Diabetes Institute, University of Alberta, 6126 HRIF East, Edmonton, AB T6G 2E1, Canada

e-mail: mraja@pmcol.ualberta.ca
}

\section{Introduction}

To maintain the barrier properties and the functioning of a biological membrane, a given membrane dynamics is required. Concerning barrier properties, the dynamics of membrane lipids and lipid-protein interactions is thought to be more important. Biological functioning is assured by the dynamics of the membrane proteins, which also depend on lipid-protein interactions (Dowhan 1997). The balance between lipid and protein dynamics and the role of lipidprotein interaction in maintaining membrane functionality are still not fully understood.

Biological membranes contain a diversity of phospholipids, which differ in the nature of their headgroups. Phosphatidic acid (PA) is a unique acidic phospholipid because of its small, negatively charged headgroup very close to the acyl chain region of the bilayer; its high affinity for divalent cations; and its propensity to form intermolecular hydrogen bonds (Demel et al. 1992; Boggs 1987). PA is not an abundant lipid constituent of any living organism, but it is extremely important as an intermediate in the biosynthesis of triacylglycerols and phospholipids and as a signaling molecule. It is the simplest diacylglycerophospholipid and the only one with a phosphomonoester as the headgroup.

The biosynthesis of another unique phospholipid, cardiolipin (CL), begins with the formation of PA from glycerol-3-phosphate and activated fatty acids. CL is a structurally dimeric phospholipid localized in the inner mitochondrial membrane, where it is required for optimal mitochondrial function. For instance, in mitochondria, a certain portion of CL is bound to proteins (Schlame et al. 1990); and in some cases, like the ADP-ATP carrier, CL is essential for the stability of the quarternary protein structure (Beyer and Nuscher 1996). 
Electrostatic interactions between anionic lipids and positively charged residues are essential for protein translocation across and insertion into the membrane (Ridder et al. 2001) and are thus responsible for the positive-inside rule (Von Heijne 1986). The question arises as to how negatively charged lipids affect protein oligomerization once they are bound to the protein surface or incorporated into protein complexes and which role they play in the overall intrinsic stability of complex assembly.

The potassium channel KcsA is an oligomeric membrane protein from Streptomyces lividans, which is a convenient model protein to study membrane protein oligomerization (Valiyaveetil et al. 2002). The tetrameric structure of KcsA is highly stable in a wide range of detergents, even in SDS (Cortes and Perozo 1997). This high stability is caused not only by interactions between protein subunits but also by interactions between the protein and the surrounding lipid bilayer (Van Dalen et al. 2002; Raja et al. 2007). In the present study, the roles of the acidic phospholipids PA and CL were investigated, with special emphasis on the involvement of the $\mathrm{N}$ terminus in determining the tetrameric stability of KcsA. The data suggest that the lipid headgroup properties of PA and CL affect channel stability differently, where the $\mathrm{N}$ terminus significantly stabilizes the tetramer via electrostatic and hydrogen bond interactions between PA and basic residues. In contrast, the interaction between $\mathrm{CL}$ and the $\mathrm{N}$ terminus plays a minor role in channel stability, thus indicating that CL might stabilize the tetramer via its exclusive interaction with positively charged residues at protein subunit interfaces of tetrameric assembly.

\section{Experimental Procedures}

\section{Reagents}

1,2-Dioleoyl-sn-glycero-3-phosphocholine (DOPC), 1,2-dioleoyl-sn-glycero-3-phosphatidic acid (DOPA) and 1, $1^{\prime}$, 2,2'-tetraoleyl cardiolipin (TOCL) were purchased from Avanti Polar Lipids (Birmingham, AL). n-Dodecyl$\beta$-D-maltoside (DDM) was from Anatrace (Maumee, $\mathrm{OH}$ ). $\mathrm{Ni}^{2+}$-NTA agarose was obtained from Qiagen (Chatsworth, CA). 2,2,2-Trifluoroethanol (TFE) was obtained from Merck (Darmstadt, Germany).

Protein Purification and Reconstitution into Proteoliposomes

Wild-type (WT) and mutant-lacking the first 18 amino acids $(\Delta 1-18)$, named " $\Delta \mathrm{N}-\mathrm{Kcs} \mathrm{A}$ "-were expressed with an N-terminal His-tag from pT7-KcsA in Escherichia coli strain BL21 according to the method described previously
(Raja et al. 2007). WT and $\Delta \mathrm{N}-\mathrm{KcsA}$ were purified with yields of $\sim 1$ and $\sim 0.1 \mathrm{mg} / \mathrm{l}$ culture, respectively. Proteins were reconstituted in different lipid mixtures with a 1:1,000 protein:lipid molar ratio, and the resultant proteoliposomes were obtained as described previously (Raja et al. 2007).

\section{Stability Assay of Tetramer Dissociation by TFE}

Small aliquots of proteoliposomes were incubated with variable concentrations of TFE for $1 \mathrm{~h}$ at room temperature, run on $15 \%$ SDS gel and analyzed by silver nitratestained SDS-PAGE. The amount of tetramer (\%) was determined and plotted against TFE (vol.\%) for the stability assay, as described previously (Raja et al. 2007).

\section{Results}

Interaction of PA and Tetramer Dissociation

Figure 1a illustrates silver-stained gels of WT and $\Delta \mathrm{N}-\mathrm{Kcs}$ A dissociation in PC:PA (7:3 mol\%) lipid bilayer as a function of TFE (vol.\%). Dissociation of WT-KcsA tetramer, which runs at $\sim 68 \mathrm{kDa}$, required relatively higher amounts of TFE (vol.\%); i.e., 50 vol.\% TFE was required to dissociate tetramer into its monomeric subunits, which run at $\sim 18 \mathrm{kDa}$, as also reported previously (Raja et al. 2007). Furthermore, $\Delta \mathrm{N}-\mathrm{KcsA}$, which still forms a stable tetramer upon $\mathrm{N}$-terminal deletion, required almost similar amounts of TFE to dissociate the tetramer. Surprisingly, tetramer dissociation in $\Delta \mathrm{N}-\mathrm{KcsA}$ could not be followed by the appearance of a monomeric population as observed for WT-KcsA dissociation. This suggests that PA specifically interacts with the $\mathrm{N}$ terminus and might be required for proper folding of monomeric subunits upon TFE-induced tetramer dissociation.

Figure $1 \mathrm{~b}$ summarizes the data from the experiments illustrated in Fig. 1a. The stability of the tetramer was found to be similar for both WT and $\Delta \mathrm{N}-\mathrm{K} c s \mathrm{~A}$ since similar amounts of TFE (vol.\%) were required to dissociate the tetramer. However, the amount of tetrameric fraction (\%) of $\Delta \mathrm{N}-\mathrm{Kcs} \mathrm{A}$ significantly decreased, in the range of 25-35 vol.\% TFE, compared to WT-KcsA. These results indicate the role of the $\mathrm{N}$ terminus in stabilizing the KcsA tetramer via specific interaction with PA.

Interaction of CL and KcsA Stability

Gel images of TFE-induced tetramer dissociation of WT and $\triangle \mathrm{N}-\mathrm{KcsA}$ in PC:CL $(7: 3 \mathrm{~mol} \%)$ lipid bilayer are shown in Fig. 2a, and Fig. 2b summarizes the data from gel electrophoresis illustrated in Fig. 2a. The amount of TFE 

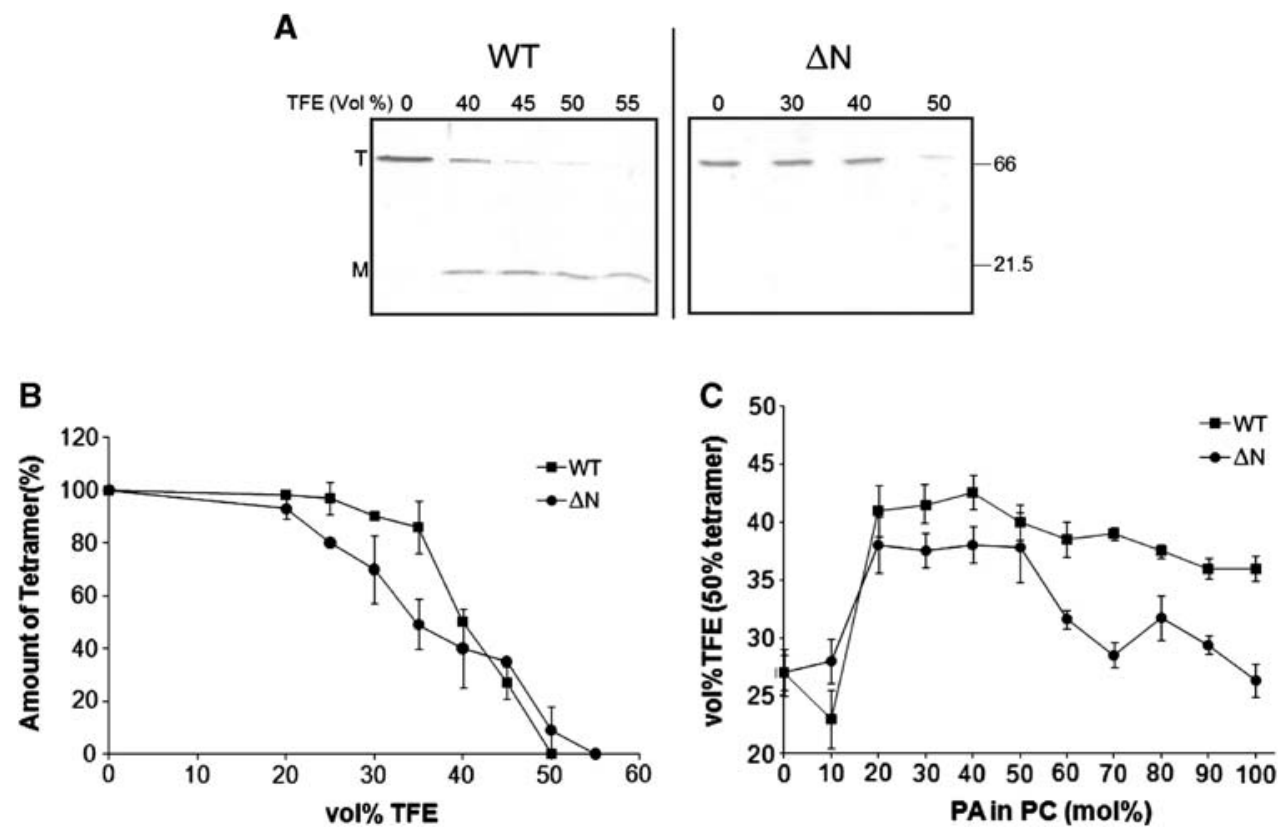

Fig. 1 a Silver-stained gels of TFE-induced tetramer dissociation in WT and $\Delta \mathrm{N}-\mathrm{KcsA}$ in PC:PA (7:3 mol\%) lipid bilayers. Tetrameric $(T)$ and monomeric $(M)$ KcsA are indicated, and a protein marker (in $\mathrm{kDa}$ ) is shown on the right. b Quantification of silver-stained gels shown in a. The intensities of tetramer bands were assigned as a relative value of $100 \%$ observed for a TFE untreated ( 0 vol. $\%$ TFE)

sample. c Effect of PA on tetramer stability of WT and $\Delta \mathrm{N}-\mathrm{Kcs}$. Proteins were reconstituted in LUVs containing varying amounts of PA in PC $(\mathrm{mol} \%)$. Samples were analyzed as described in $\mathbf{b}$, and the vol. $\%$ TFE required to dissociate the $50 \%$ tetramer was calculated. All data points correspond to the average \pm SD of three experiments

Fig. 2 a Silver-stained gels of TFE-induced tetramer dissociation in WT and $\Delta \mathrm{N}-\mathrm{KcsA}$ in PC:CL $(7: 3 \mathrm{~mol} \%)$ lipid bilayers. Tetrameric $(T)$ and monomeric $(M)$ KcsA are indicated, and a protein marker (in $\mathrm{kDa}$ ) is shown on the right. $\mathbf{b}$ Quantification of silver-stained gels shown in a. The \% tetramer was calculated as described for Fig. 1b. $\mathbf{c}$ Influence of CL on tetrameric stability of WT and $\Delta \mathrm{N}-\mathrm{KcsA}$ in PC bilayers. The amount of $50 \%$ tetramer was calculated as described for Fig. 1c. All data points correspond to the average $\pm \mathrm{SD}$ of three experiments
A
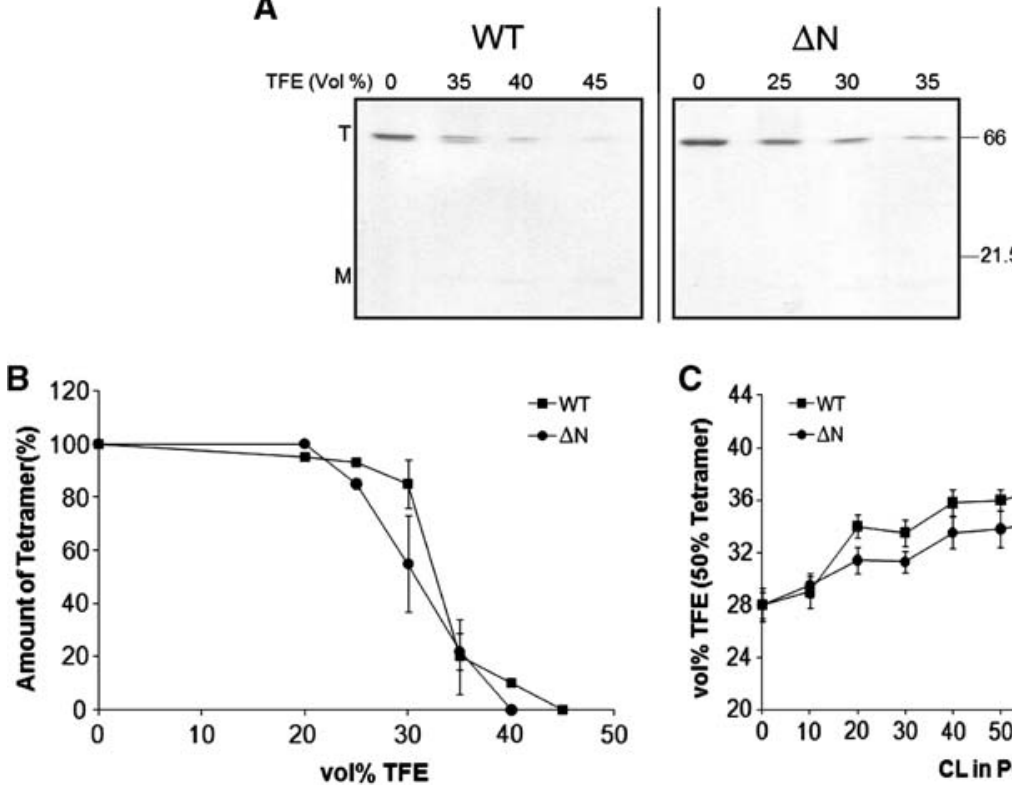

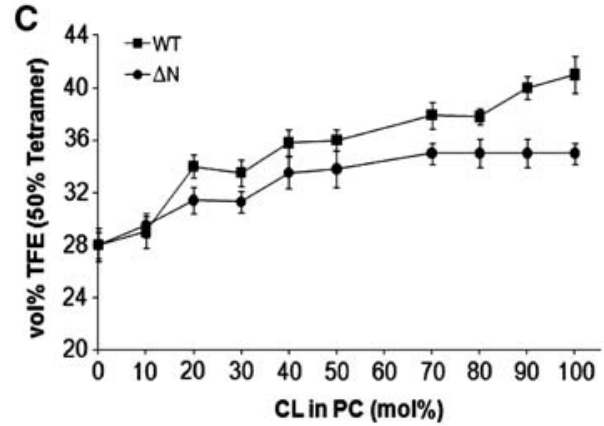

(vol.\%) required to dissociate both tetrameric proteins was found to be identical, thus exhibiting similar stability. Furthermore, no significant difference in TFE-induced remaining tetrameric fraction $(\%)$ was observed. It is also interesting to note that upon tetramer dissociation of both proteins no monomeric KcsA could be observed on SDS gel, which could be due to aggregation of the monomeric KcsA-CL complex.
Influence of PA and CL on the Stability of Tetrameric Assembly

The interaction of PA and CL with KcsA and their roles in stabilizing tetrameric structure were further analyzed by titrating an increasing amount of PA or CL in PC bilayers, as shown in Figs. 1c and 2c, respectively. The amount of TFE (vol.\%) at which $50 \%$ tetramer was stable was 
determined as a function of anionic PA or CL present in the membrane. For WT-KcsA, the tetramer is stabilized in the range of $20-50 \mathrm{~mol} \% \mathrm{PA}$ in $\mathrm{PC}$ compared to pure $\mathrm{PC}$ (0 mol\% PA) (Fig. 1c). Increasing PA from 60 to $100 \mathrm{~mol} \%$ leads to a slight decrease in tetrameric stability, possibly due to a high charge density in the membrane, which seems to affect protein-lipid interaction. In case of $\Delta \mathrm{N}-\mathrm{Kcs} \mathrm{A}$, tetrameric stability was slightly reduced, in the range of $20-50 \mathrm{~mol} \% \mathrm{PA}$ in PC; however, a significant reduction was observed at higher PA concentrations compared to WT-KcsA. These results suggest that the $\mathrm{N}$ terminus has a specific interaction with PA and this interaction seems to increase tetrameric stability. It is also interesting to note that the $\mathrm{N}$ terminus significantly compensates for the destabilizing effect of high charge density in the membrane.

On the other hand, the stability of WT-KcsA significantly increased throughout the range of CL, indicating that saturation or an optimal CL content required to occupy all binding sites of KcsA could not be reached (Fig. 2c). However, in the absence of the $\mathrm{N}$ terminus, the optimal CL-KcsA interaction was reached at $50 \mathrm{~mol} \% \mathrm{CL}$ in lipid bilayers. These results suggest that the two anionic lipids interact with KcsA differently. PA significantly stabilizes the tetramer via its interaction with the $\mathrm{N}$ terminus. The interaction of CL with the $\mathrm{N}$ terminus does not seem to be important for stabilizing the tetrameric structure, thus suggesting that CL might stabilize the tetramer via specific protein-lipid interactions at protein subunit interfaces (see "Discussion" section).

\section{Discussion}

The formation of homo-oligomeric assemblies is a wellestablished characteristic of many soluble proteins and enzymes. Oligomerization has been shown to increase protein stability, allow allosteric cooperativity and provide multivalent interaction sites in soluble proteins. In comparison, our understanding of the prevalence and the reasons behind protein oligomerization and stability in oligomeric membrane proteins is relatively insufficient. The present study investigated channel stability, with special emphasis on the role of the $\mathrm{N}$ terminus, which forms an alpha-helix anchored at the membrane-water interface (Cortes et al. 2001). The $\mathrm{N}$ terminus contains two arginines, at positions 11 and 19, and one lysine, at position 14. It was interesting to observe a stronger stabilizing effect of PA than CL. In addition, the $\mathrm{N}$ terminus significantly contributed to the stabilizing effect of PA. Previous studies also indicated that PA has a stronger stabilizing effect than anionic phosphatidylglycerol (PG) (Raja et al. 2007). However, it should be noted that the phosphomonoester headgroup of PA has remarkable H-bonding properties (Kooijman et al. 2003). It has been demonstrated that the positively charged lysine and arginine residues on proteins can bind with some specificity to PA through hydrogen bonding with the phosphate group, thus distinguishing PA from other phospholipids. An "electrostatic-hydrogen bond switch model" has been proposed in which the headgroup of PA forms a hydrogen bond to a basic amino acid residue, leading to deprotonation of the headgroup, increasing its negative charge and thus enabling stronger interactions with further basic residues (see schematic model in Fig. 3a) and tight docking with the membrane interacting protein (Kooijman et al. 2007; Raja et al. 2007). Furthermore, TFE also strengthens the electrostatic-hydrogen bond switch between the basic residues and PA since it promotes ionic interactions in soluble proteins (Raja et al. 2007).

It is surprising that PA, which contains a net charge of $\sim 1.5$ (Demel et al. 1992), still exhibits a stronger stabilizing effect than CL, which potentially carries two negative charges (LeCocq and Ballou 1964). In this context, the lipid headgroup properties of PA can be taken into further consideration. PA behaves as a cone-shaped lipid similar to the molecular shape of zwitterionic phosphatidylethanolamine (PE) (Kooijman et al. 2007). PE has been shown to increase membrane association and facilitate insertion of monomeric KcsA due to its small headgroup size (Ridder et al. 2001). Similarly, the small size of the PA headgroup might also allow efficient and deep insertion of the KcsA subunit and $\mathrm{N}$ terminus into the membrane, thus increasing channel stability via H-bonding/electrostatic interactions. Furthermore, a slight stabilizing effect of PG observed in the previous study (Raja et al. 2007) was found to be specific due to electrostatic interaction between the N-terminal helix dipole and the acidic PG, and this assumption was supported by the observation that high salt concentration in the reaction medium diminished the strength of ionic binding by providing competing ions for the charged residues, thus abolishing the PG-dependent stabilizing effect (unpublished data). There is a possibility of binding of His-tag to acidic phospholipids. Such an effect does not, however, contribute to the stabilizing differences between the two proteins. The reason is that channel stability is compared in the presence or absence of the $\mathrm{N}$-terminal domain and in both cases the His-tag was present, which facilitates a more straightforward comparison of the two proteins.

It is clear that the amount of TFE required to dissociate the KcsA-PG complex, as observed previously (Raja et al. 2007), is relatively less than that required for dissociation of the KcsA-CL complex, thus indicating that an increase in net charge promotes strong electrostatic interactions between basic residues and the CL headgroup. Such interactions, however, do not seem as powerful as 
A

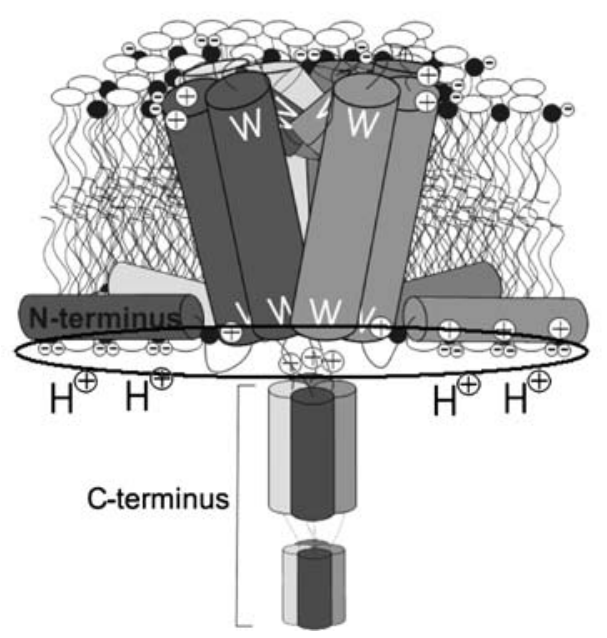

Fig. 3 A schematic model of KcsA interaction with PA and CL in a lipid bilayer. a The interaction of PA (black small headgroup) with the $\mathrm{N}$ terminus and positively charged region (R27, R117, R121 or R122), as suggested previously (Raja et al. 2007), has been indicated at the cytoplasmic side. b The interaction of CL (black headgroups) with positively charged residues (R64, R89 or R52) at subunit

electrostatic-hydrogen bond interactions in the PA-KcsA complex. Furthermore, the $\mathrm{N}$ terminus does not have a significant role in stabilizing the KcsA-CL complex, which indicates that $\mathrm{CL}$ might stabilize the tetrameric structure differently from PA.

Further insight into the nature of CL-protein interactions may be derived from crystal structures of CL-protein complexes. Tightly bound CL has been identified in crystals of mitochondrial complex III (Lange et al. 2001) and the ADP-ATP carrier (Pebay-Peyroula et al. 2003), as well as in crystallized prokaryotic proteins, such as the trimeric formate dehydrogenase $N$ (Jormakka et al. 2002) and succinate dehydrogenase (Yankovskaya et al. 2003). In all these protein crystals, the headgroup of CL forms strong hydrophilic interactions with a number of amino acid residues, involving electrostatic forces, hydrogen bonds and water molecules. The acyl chains, however, remain flexible and interact with the protein surface by van der Waals forces at multiple sites. CL has been shown to sit typically at monomer interfaces of oligomeric assemblies and appears to mediate the contact between two monomers or the contact between the protein surface and the bilayer.

It can be assumed that $\mathrm{CL}$ also sits at monomer interfaces of tetrameric assembly in the close proximity of R64 and R89 (see Fig. 3b), which are known to be potential binding sites for PG, as suggested from the KcsA-PG crystal complex (Valiyaveetil et al. 2002). In this regard, R52 would also be involved in such interactions because its side chain sticks out toward the membrane interface (Doyle et al. 1998) and might interact with CL. The importance of

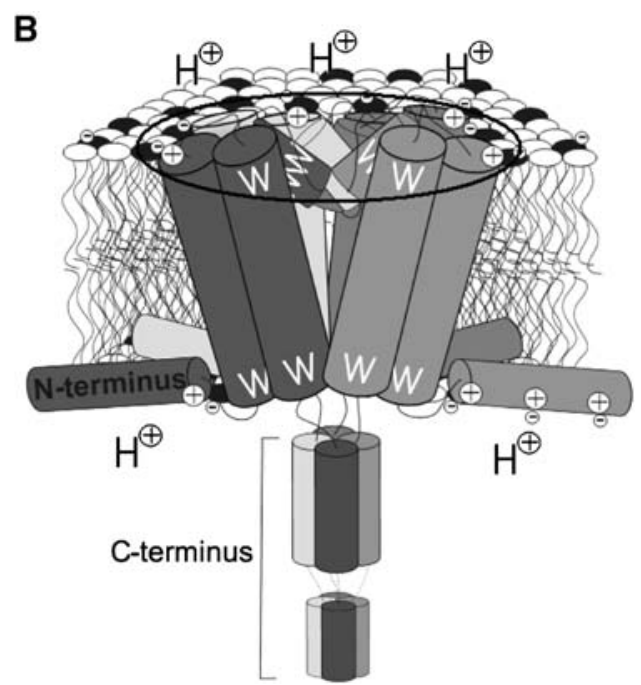

interfaces of KcsA has been proposed. The positions of Trp $(W)$ residues at membrane-water interfacial regions are also indicated. Deprotonation of anionic headgroups upon binding of side chains of basic residues (Arg and/or Lys) enhances electrostatic interaction, thereby stabilizing the protein-lipid interaction

a CL-induced stabilizing effect can also be justified by the fact that it plays a role in the formation of supercomplexes because CL deficiency decreases the stability of large supramolecular aggregates (Zhang et al. 2002; McKenzie et al. 2006).

Acknowledgements The construct of N-terminally deleted $(\Delta \mathrm{N})$ KcsA was a precious gift from the late Jeanette G. Stam. The careful secretarial help of Irene van Duin and helpful suggestions of Antoinette Killian and Ben de Kruijff (Department of Biochemistry of Membranes, Utrecht University) are gratefully acknowledged. This work was supported by funds of the Chemical Sciences Division of the Netherlands Organization for Scientific Research.

Open Access This article is distributed under the terms of the Creative Commons Attribution Noncommercial License which permits any noncommercial use, distribution, and reproduction in any medium, provided the original author(s) and source are credited.

\section{References}

Beyer K, Nuscher B (1996) Specific cardiolipin binding interferes with labeling of sulfhydryl residues in the adenosine diphosphate/adenosine triphosphate carrier protein from beef heart mitochondria. Biochemistry 35:15784-15790

Boggs JM (1987) Lipid intermolecular hydrogen bonding. Influence on structural organization and membrane function. Biochim Biophys Acta 906:353-404

Cortes DM, Perozo E (1997) Structural dynamics of the Streptomyces lividans $\mathrm{K}^{+}$channel (Skcl): oligomeric stoichiometry and stability. Biochemistry 36:10343-10352

Cortes DM, Cuello LG, Perozo E (2001) Molecular architecture of full-length KcsA: role of cytoplasmic domains in ion permeation and activation gating. J Gen Physiol 117:165-180 
Demel RA, Yin CC, Lin BZ, Hauser H (1992) Monolayer characteristics and thermal behaviour of phosphatidic acids. Chem Phys Lipids 60:209-223

Dowhan W (1997) Molecular basis for membrane phospholipid diversity: why are there so many lipids? Annu Rev Biochem 66:199-232

Doyle DA, Morais CJ, Pfuetzner RA, Kuo A, Gulbis JM, Cohen SL, Chait BT, MacKinnon R (1998) The structure of the potassium channel: molecular basis of $\mathrm{K}^{+}$conduction and selectivity. Science 280:69-77

Jormakka M, Tornroth S, Byrne B, Iwata S (2002) Molecular basis of proton motive force generation: structure of formate dehydrogenase N. Science 295:1863-1868

Kooijman EE, Chupin V, de Kruijff B, Burger KNJ (2003) Modulation of membrane curvature by phosphatidic acid and lysophosphatidic acid. Traffic 4:162-174

Kooijman EE, Tieleman DP, Testerink C, Munnik T, Rijkers DTS, Burger KNJ, de Kruijff B (2007) An electrostatic/hydrogen bond switch as the basis for the specific interaction of phosphatidic acid with proteins. J Biol Chem 282:11356-11364

Lange C, Nett JH, Trumpower BL, Hunte C (2001) Specific roles of protein-phospholipid interactions in the yeast cytochrome $\mathrm{bc}_{1}$ complex structure. EMBO J 20:6591-6600

LeCocq J, Ballou CE (1964) On the structure of cardiolipin. Biochemistry 3:976-980

McKenzie M, Lazarou M, Thorburn DR, Ryan MT (2006) Mitochondrial respiratory chain supercomplexes are destabilized in Barth syndrome patients. J Mol Biol 361:462-469

Pebay-Peyroula E, Dahout G, Kahn R, Trezeguet V, Lauquin GJ, Brandolin G (2003) Structure of mitochondrial ADP/ATP carrier in complex with carboxyatractyloside. Nature 426:39-44
Raja M, Spelbrink REJ, de Kruijff B, Killian JA (2007) Phosphatidic acid plays a special role in stabilizing and folding of the tetrameric potassium channel KcsA. FEBS Lett 581:5715-5722

Ridder ANJA, Kuhn A, Killian JA, de Kruijff B (2001) Anionic lipids stimulate sec-independent insertion of a membrane protein lacking charged amino acid side chains. EMBO Rep 2:403-408

Schlame M, Horvath L, Vigh L (1990) Relationship between lipid saturation and lipid-protein interaction in liver mitochondria modified by catalytic hydrogenation with reference to cardiolipin molecular species. Biochem J 265:79-85

Valiyaveetil FI, Zhou Y, MacKinnon R (2002) Lipids in the structure, folding, and function of the $\mathrm{KcsA} \mathrm{K}^{+}$channel. Biochemistry 41:10771-10777

Van Dalen A, Hegger S, Killian JA, de Kruijff B (2002) Influence of lipids on membrane assembly and stability of the potassium channel KcsA. FEBS Lett 525:33-38

Von Heijne G (1986) The distribution of positively charged residues in bacterial inner membrane proteins correlates with the transmembrane topology. EMBO J 5:3021-3027

Yankovskaya V, Horsefield R, Tornroth S, Luna-Chavez C, Miyoshi H, Leger C, Byrne B, Cecchini G, Iwata S (2003) Architecture of succinate dehydrogenase and reactive oxygen species generation. Science 299:700-704

Zhang M, Mileykovskaya E, Dowhan W (2002) Gluing the respiratory chain together. Cardiolipin is required for supercomplex formation in the inner mitochondrial membrane. J Biol Chem 277:43553-43556 